\title{
Culturas tradicionales y cambios contemporáneos: el pueblo indígena kokonuco y las tecnologías de la información y la comunicación
}

\author{
PAOLA ANDrea Acosta NATES*
}

Artículo recibido: 27 de febrero de 2014

Artículo aprobado: 9 de abril de 2014

Doi: dx.doi.org/10.12804/desafios26.02.2014.06

Para citar este artículo: Acosta Nates, P. A. (2014). Culturas tradicionales y cambios contemporáneos: el pueblo indígena kokonuco y las tecnologías de la información y la comunicación. Desafíos, 26 (2), 153-178. doi: dx.doi.org/10.12804/desafios26.02.2014.06

\section{Resumen}

La inclusión de las tecnologías de la información y de la comunicación (TIC) en pueblos indigenas se extiende mediante diferentes proyectos y estrategias que el Estado ha formulado para promover así la generalización y su uso bajo una política de "inclusión". En Colombia, los pueblos indígenas han optado por diversas estrategias para incorporar las TIC de manera diferencial en sus formas de vida; cambio contemporáneo que ha posibilitado desarrollar paulatinamente vínculos político-sociales y comunicacionales más allá de la dimensión territorial física. Por medio de la antropología de la tecnología $y$, particularmente, desde la etnografía se describen en este texto las redes sociocomunicativas que ha generado internety se analizan, a su vez, los modelos de apropiación gradual de la tecnología en el pueblo indígena kokonuco, ubicado en el departamento

\footnotetext{
* Antropóloga y magíster en Antropología Crítica, egresada de la Universidad del Cauca, Colombia. Integrante del grupo de investigación Territorialidades de la Universidad de Caldas, Colombia. Correo electrónico: paonates@gmail.com
} 
del Cauca, Colombia. Lo anterior con el interés de construir transversalmente el problema del uso y apropiación de las TIC en pueblos indígenas.

Palabras clave: TIC, redes sociales, cambios contemporáneos, comunicación, modelo.

\title{
Traditional Cultures and Contemporary Changes: Kokonuco Ethnic People and Information and Communication Technologies (ICTS)
}

\begin{abstract}
Under a policy of "inclusion", the state has made a generalized effort to promote the spread of ICTs within indigenous peoples, using different projects and strategies. In Colombia, indigenous peoples have chosen different strategies in order to incorporate ICTs into their lifestyles, which, in turn, has enabled gradual change to develop social policy and communication links beyond the territorial dimension. The model of the gradual appropriation of technology within the Kokonuco indigenous peoples located in the Cauca region of Colombia is analyzed through an anthropology of technology and particularly through the ethnography of socio-communicative internet networks. The concern with the problem of the use and appropriation of ICTs in indigenous peoples tries to answer the following question: How are ICTs perceived, socially appropriated and re-signified in the Kokonuco indigenous peoples?
\end{abstract}

Keywords: Technology, social networking, change, communication.

\section{Culturas tradicionais e mudanças contemporâneas: o povo indígena kokonuco e as tecnologias da informação e a comunicação}

\begin{abstract}
Resumo
A inclusão das TIC em povos indígenas estende-se através de diferentes projetos e estratégias que o Estado tem formulado de modo generalizado para promover assim a generalização e seu uso sob uma política de "inclusão". Na Colômbia os povos indígenas têm optado por diversas estratégias para incorporar as TIC de forma diferencial em suas formas de vida; mudança contemporânea que tem possibilitado desenvolver paulatinamente vínculos político-sociais e comunicacionais além da dimensão territo-
\end{abstract}


rial física. Através da antropologia da tecnologia e particularmente desde a etnografia, descrevem-se neste texto as redes sócio-comunicativas que tem gerado a Internet $e$ analisam-se igualmente os modelos de apropriação gradual da tecnologia no povo indígena kokonuco localizado no departamento do Cauca na Colômbia. O anterior com o interesse de construir transversalmente o problema do uso e apropriação das TIC em povos indígenas.

Palavras chave: TIC, redes sociais, mudanças contemporâneas, comunicação, modelo.

\section{Introducción}

En años recientes, las tecnologías de la información y la comunicación (TIC) y la sociedad de la información se han convertido en categorías agenciadas por el Estado y organismos multilaterales internacionales que buscan integrar a grupos humanos bajo el "pretexto" de reducir la denominada brecha digital. En el fondo, los desarrollos tecnológicos han sido producto de una necesidad inducida por las fuerzas del mercado que, efectivamente, han integrado gradualmente diversos grupos humanos y, entre ellos, las poblaciones indígenas. Dispositivos y aplicaciones como computadores, internet, MP5, tablets, memorias micro de alta capacidad, entre otros, son ejemplos materiales que en la década de los noventa orientaron la mirada de la tecnología a la ciencia y el desarrollo y que proyectaron dicha perspectiva hacia la sociedad y la cultura.

En el ámbito mencionado, las TIC — particularmente internet — han cumplido actualmente (2014) un papel central para muchos pueblos indígenas, en cuanto a la validación de sus proyectos o planes de vida, ${ }^{1}$

\footnotetext{
1 Los planes de vida se han ido ampliando en el continente americano. Algunos pueblos en Canadá y Paraguay los han formulado (Sandt, 2012). En el caso de Colombia, fue adoptado por el Consejo Regional Indígena del Cauca (Cric), en sustitución de los planes de desarrollo. Para algunos autores como Gow se trata de "una estrategia a largo plazo para el desarrollo integral del resguardo y toma en cuenta todos los aspectos de la sociedad y la cultura indígena, presentando una visión para el futuro y contestando implícitamente las siguientes preguntas: ¿quiénes somos?, ¿de dónde venimos?, ¿a dónde vamos?” (2010, p. 115).
} 
lo que ha planteado diversas posibilidades de uso y apropiación. Para la población indígena kokonuco, ubicada en el departamento del Cauca, al sur de Colombia, internet ha resultado ser un importante medio para procesar, difundir, limitar y generar un sinnúmero de procesos relacionados con temáticas como unidad, autonomía, organización, comunicación, territorio, economía y política que, hoy por hoy, les ha permitido desarrollar paulatinamente vínculos político-sociales y comunicativos más allá de la dimensión territorial física.

Ahora bien, para aprehender el fenómeno en el contexto teóricometodológico de la antropología de la tecnología ${ }^{2}$ y la práctica citada se trabajaron tres categorías: tecnología, redes sociales y modelos, a la luz de los pueblos indígenas. Del mismo modo, se consideraron metodológicamente dos vertientes para rastrear los nexos entre el mundo digital y el espacio físico de la población indígena kokonuco con respecto a uso y apropiación de las tecnologías: una dimensión on line y otra en offline (Ardèvol, 2003), unidades convergentes por medio de las cuales se buscó responder a las distintas dimensiones en las que las TIC son percibidas, apropiadas y resignificadas en la población indígena kokonuco, tema que se pondera en la primera sección de este artículo.

Posteriormente se aborda una aproximación a dos experiencias que el pueblo kokonuco, en particular el resguardo de Puracé, ha tenido en cuanto al uso y manejo de las TIC. A partir de esta experiencia se rescatan los conceptos propuestos, especialmente los de modelos y redes, que en este caso fueron ejemplarizantes para ilustrar la dinámica que se revelaba en este contexto. Finalmente, y a manera de conclusión, se presentan algunas consideraciones sobre la relación uso, apropiación de las TIC y pueblos indígenas.

\footnotetext{
2 Enfoque que, según Tocancipá-Falla (2013 en edición), estudia las interrelaciones que se establecen entre tecnología, sistemas socioculturales y el medio. La diferencia entre antropología de la tecnología y los estudios de ciencia y tecnología transita en su instrumentalidad. Arturo Escobar llama la atención hacia pensar el tema de la tecnología desde posturas críticas de enunciación y producción (2005, citado en Cabrera, Moriones y Quinchoa, 2003). Lemonnier (1996), por su parte, deja entrever la existente correspondencia entre objetos, actividades, procesos, conocimientos y habilidades en su perspectiva de tecnología.
} 


\section{La tecnología, redes y modelos como categorías analíticas}

En las ciencias sociales, y en particular en la antropología de la tecnología, la reflexión teórica relacionada con los conceptos de tecnología, redes, comunicación y modelos constituye un debate prolífico que va más allá del acceso y del uso y se vincula con la vital importancia de tener claro cómo es que lo sociocultural va incursionando en los procesos tecnológicos y qué factores, aspectos y actores influyen en ello. En este sentido, la aproximación teórica a estos cambios se ha dado desde enfoques planteados en los estudios de ciencia, tecnología y sociedad, la antropología (e. g. "La antropología de la cibercultura", de Arturo Escobar, o la "Antropología de la tecnología", de Pierre Lemmonier) y la misma sociología de la ciencia.

El antropólogo colombiano Arturo Escobar (2005), en su postura devenida de una tradición culturalista estadounidense, dejaba entrever la importancia de indagar sobre la tecnología y sus reflejos recíprocos en la cultura; aunque la idea de sociedad estaba latente, se centró más en una idea constructivista de "cultura", propia de dicha tradición. Para él la cibercultura es "un campo de fuerzas y significados [... donde la] compleja producción de sentidos de vida, de trabajo y de lenguaje, toma lugar" (Escobar, 2005, p. 22). Esta definición es consecuente con su valoración de que la tecnología no es solo el resultado de una "nueva cuadrícula de control en el planeta", sino que también plantea un conjunto de "nuevas posibilidades para articulaciones potenciales entre los seres humanos, la naturaleza y las máquinas" (Escobar, 2005, p. 22).

Por otra parte, el trabajo llamado "Technology", de Pierre Lemonnier (1996), permite apreciar valores interpretativos de diferente índole, aunque convergentes en otras con el trabajo de Escobar. Por ejemplo, Lemonnier, al igual que Escobar, coincide en que uno de los centros de atención es el valor de significado y de representación que los grupos le confieren a la tecnología, valor que en Lemmonier está centrado en lo simbólico. 
Un aporte similar sobre la dimensión simbólica, de las representaciones y de procesos mentales, es planteado por el antropólogo Arjun Apadurai (2001), en su texto La modernidad desbordada, donde expone estas dimensiones culturales de la globalización y propone pensar el mundo globalizado bajo la premisa de un "trabajo de la imaginación"; pensada como el lugar donde los individuos y los grupos negocian y disputan simbólicamente cómo anexar lo global a sus prácticas. A pesar de estos inicios teóricos en la antropología, y aparte del trabajo teórico de Appadurai, Escobar y Lemonnier, no se ha examinado ni problematizado lo suficientemente la relación existente entre el papel de las "nuevas" TIC, en especial internet, y las poblaciones campesinas, afros y mucho menos en poblaciones indígenas en Colombia.

Por ello, en principio, estas aproximaciones teóricas nos permiten dar cuenta de las interrelaciones y modelos que se producen con el uso y apropiación o no de la tecnología por poblaciones indígenas y cómo estas han respondido social y culturalmente a dichos procesos. En esta dirección, la temática se convierte en un desafío permanente para la antropología y las ciencias sociales, pues cada vez más las TIC adquieren una dimensión ambigua que evoca viejas discusiones sobre el papel de la tecnología en la humanidad (Cabrera, Moriones y Quinchoa, 2003; Castell, 1999; Escobar, 2005; Lemonnier, 1996; Woolgar, 1996). Por ejemplo, el carácter inocuo e inofensivo que le atribuye el Ministerio de Telecomunicaciones revela esa visión neutral. Así, las TIC son definidas como "el conjunto de herramientas, equipos, programas informáticos, aplicaciones, redes y medios que permiten la compilación, procesamiento, almacenamiento, trasmisión de información como: voz, datos, texto, video e imágenes" (Ministerio de Comunicaciones de Colombia, 2008, p. 4; Moriones, 2011).

Manuel Medina sugiere igualmente una idea más integrante y vinculante más allá del objeto o artefacto material:

No solo capacidades, actividades y realizaciones de carácter simbólico (tales como representaciones e interpretaciones simbólicas, discursivas, artísticas, teóricas, cosmovisivas [sic], valorativas, etc., 
es decir, la cultura en su acepción más registrada), sino también técnicas y artefactos materiales, formas organizativas de interacción social, económica, territorial y política (lo que se entiende económicamente por sociedad) y prácticas y realizaciones biotécnicas, relacionadas con los seres vivos y el entorno biótico (o naturaleza en sentido general) (2003, p. 44).

De aquí se entiende que cualquier práctica cultural y social implica la conexión de los diversos aspectos mencionados; es decir, las prácticas están mediadas por artefactos materiales, y estas, de alguna forma, son representadas e interpretadas simbólicamente, articuladas socialmente y contextualizadas ambientalmente. De ahí que estos aspectos no son entidades separadas y disociadas, sino que están en continuo dinamismo y conexión, tema que se aborda en la siguiente sección.

\section{La dimensión relacional y articuladora (red) de las TIC}

Cualquier práctica cultural y social implica la conexión de diversos aspectos. Cuestionarnos sobre este dinamismo en la población indígena kokonuco implica la reconsideración de procesos externos y la reconfiguración de procesos internos para poder establecer negociaciones y relaciones con diversos actores sociales. $\mathrm{Al}$ respecto, Ulloa refiere a la denominada autonomía relacional indígena, que según, en el contexto actual de los pueblos indígenas es entendida:

Como la capacidad de los pueblos indígenas para ejercer autodeterminación y gobernabilidad en sus territorios a partir de las relaciones, negociaciones, confrontación y participación que tienen que establecer con el estado [sic] y diversos actores locales, nacionales y transnacionales; aun cuando retomen políticas o procesos estatales o transnacionales en la búsqueda de su consolidación. De igual manera, en aras del reconocimiento deben ajustarse a los planes de desarrollo nacionales y a las lógicas del desarrollo en cuanto a planificación, conocimientos expertos y mejoramiento de condiciones de vida y cambio de prácticas productivas. En estos contextos las fronteras conceptuales, políticas y territoriales están en permanente reconfiguración (2012, p. 76). 
Para el pueblo indígena kokonuco la autonomía relacional indígena en el tema de las TIC implica su capacidad de relacionarse con otras instituciones y actores que pueden catalizar o fortalecer sus propuestas de educación, políticas, territorio, etc., de acuerdo con sus visiones, necesidades (pasadas, presentes o de futuro, integradas en sus planes de vida) y prácticas culturales. Otro punto de vista que complementa teóricamente la perspectiva anterior es la teoría del actor red (TAR). Latour (2005) propone una serie de estrategias diversas bajo el supuesto de que se puede "trazar" y establecer una red que comunique los aspectos sociales que conectan a los actores entre sí, las tecnologías, su impacto en la sociedad y las nuevas prácticas que aparecen con el origen y el proceso de elaboración de dichas tecnologías. Así, desde este planteamiento social, ya no se referiría a una cosa en medio de otras, sino a un tipo de conexión entre cosas que no son, ellas mismas, estrictamente sociales pero que tienen al mismo tiempo un impacto social.

\section{La categoría de modelo en las TIC}

Junto a la perspectiva de red, la categoría de modelo es un complemento, pues evidencia cómo se configura una práctica cultural que viene dada por un conjunto de elementos diversos integrados por personas, artefactos, técnicas, interpretaciones, valoraciones, formas de organización, relaciones sociales, entre otros, "cuya afectación de uno conlleva a una implicación sobre el desempeño del otro idea asociada a la de Sistema" (Bertalanffy, 2007, p. 23; Tocancipá-Falla, 2013 en edición). Así, cada uno de los elementos que conforman determinada práctica cultural están en constitutiva dependencia de los demás, independientemente de si estos elementos tienen la cualidad de lo igual o lo homogéneo o de lo diferente o heterogéneo, planteamiento que resulta de vital importancia en el análisis relacional del papel de las TIC y de los pueblos indígenas que buscan un reconocimiento en distintas escalas (regional, nacional o internacional) y una integración, desde sus propios términos socioculturales, en marcos establecidos por el Estado.

Del mismo modo, es importante explicar algunos conceptos clave asociados con la trayectoria histórica de los pueblos indígenas en la configuración de sus modelos, como lo son el resguardo, el cabildo y 
los planes de vida. Según el plan de vida de los kokonuco, el resguardo connota un territorio delimitado por una comunidad que es indígena y que posee un título que los acredita como propietarios; idea que, además de ser reconocida en la Ley 89 de 1890, también se valida en el ámbito nacional en la Constitución Política, pues el artículo 329 atribuye su carácter de propiedad colectiva, el cual —aunado con el artículo 58 - hace referencia a la protección de la propiedad, y el artículo 63, que les confiere a estos territorios las cualidades de inalienables, imprescriptibles, inembargables (Constitución Política de Colombia, 1991).

La primera autoridad dentro de este territorio indígena es el cabildo. Se trata de:

[...] una entidad pública de derecho especial, cuyos integrantes son miembros de una comunidad Indígena, elegidos y reconocidos por esta, con una organización sociopolítica tradicional, cuya función es representar legalmente a la comunidad, ejercer la autoridad y realizar las actividades que le atribuyen las leyes, sus usos, costumbres y el reglamento interno de cada comunidad (artículo 2, Decreto 2164 de 1995) (Mazabuel, 2002, pp. 24 y 25).

El contenido principal para que el cabildo y la población indígena ejerzan su proceso de gobernabilidad en el mencionado territorio es el Plan de vida, documento que posee los lineamientos y orientaciones que constituyen la cosmovisión del pueblo kokonuco.

A partir de lo mencionado, y como veremos en los párrafos siguientes, se plantea que las prácticas tecnológicas que se llevan a cabo y que conforman las particularidades de redes y modelos en la población indígena kokonuco son más que una herramienta para beneficio y desarrollo del pueblo como tal; también son medios de comunicación y del hacer cotidiano que fortalecen los procesos de resistencia indígena frente al mismo Estado o el mercado.

El análisis de estas articulaciones conceptuales son centrales para una etnografía de cómo y qué se produce en la interrelación tecnología y 
las prácticas socioculturales que se dan en poblaciones indígenas. En esta dirección, la pregunta que se desarrolla en este artículo es: ¿cómo se resignifican y tejen las conexiones que permiten la concepción, uso, apropiación y puesta en funcionamiento de un proyecto tecnológico en la población indígena kokonuco? Tal pregunta está directamente relacionada con aspectos metodológicos, los cuales se abordan en los párrafos siguientes.

\section{Consideraciones metodológicas: ¿cómo investigar el tema tecnológico?}

El desarrollo metodológico para investigar el tema tecnológico se enmarcó en tres categorías que fueron la plataforma para la construcción teórica y metodológica del objeto de estudio (tecnología, redes y modelos), reflexión teórica que ha incorporado, gradualmente, nuevas formas de establecer relaciones con los otros.

En ese sentido, tres fases lograron ese carácter integrador. En la primera se articularon datos cualitativos y cuantitativos: se analizaron las características de la inclusión digital en poblaciones indígenas a partir de revisión de fuentes secundarias (estadísticas, informes, portales producidos tanto por grupos indígenas como por instituciones que avalan el discurso de las TIC, así como desarrollo y progreso) e igualmente se recopiló información bibliográfica acerca tecnología, redes y modelos en poblaciones indígenas. En una segunda fase se realizó una caracterización general del territorio, hecho que dio insumos para avizorar el papel que desempeña la integración y el papel articulador de este tipo de tecnologías con la vida social de los pueblos.

En una tercera fase, más en terreno del territorio kokonuco, se fueron trabajando conversatorios y talleres para identificar las estrategias de uso y apropiación, a fin de trabajar posteriormente con los indígenas en el ámbito veredal, quienes de este modo demostraban más interés y participación. Al respecto, se presentaron ejercicios de portales en texto y en imagen para reelaborarlos conjuntamente y definir las categorías y disposiciones propias del diseño. Así, y paulatinamente, se fueron generando espacios de encuentro mutuo para trabajar, que 
buscaban no solo el interés del que investiga, sino también del actor local (Rapport y Overing, 2007).

Del mismo modo, la investigación me llevó a considerar metodológicamente otras dos vertientes para rastrear los nexos entre el mundo digital y el espacio físico de la población indígena kokonuco con respecto a uso y apropiación de las tecnologías: una dimensión on line y otra en offline (Ardévol, 2003). Mientras la primera alude espacio virtual, la segunda se refiere al espacio físico. Si bien esto se plantea teórica y metodológicamente, en la práctica encontramos convergencias con lo enunciado por el actual (2013) gobernador del resguardo de Puracé, Arcadio Aguilar: "Es lo que denominaría interacciones dentro y fuera" de los mundos virtuales. Además de ser mis sitios de campo, estas dos dimensiones fueron lugares de aplicación de la etnografía, donde la triangulación de datos se fundamentó en las relaciones que establecen los actores en estos dos contextos y facilitan así una cartografía de relaciones, localizada en diversos ámbitos — tema que plantea una etnografía multisituada como lo señala Marcus (2001)—, en la que son importantes tanto las relaciones de contacto cara a cara como las virtuales.

Una de las características de esta metodología es que las relaciones que se establecieron offline en el pueblo kokonuco pasaron al entorno on line, al chatting o a Facebook. Este dinamismo permitió augurar que los pueblos indígenas van a lograr apropiar las TIC en grados diversos. Si actores sociales, como el pueblo indígena kokonuco, tienen la disposición de proyectarse a través de las TIC, queda abierto un debate entre varios puntos de reflexión que involucran el acceso y uso de las TIC como elementos conectados al plan de vida, aspecto que en las dinámicas contemporáneas reconfiguran redes que llevan a la apropiación de herramientas tecnológicas en los pueblos en cuestión.

\section{Las redes que generan apropiación de las TIC en el pueblo kokonuco}

El pueblo kokonuco hace parte de los municipios de Puracé, Coconuco, Popayán y El Tambo. La población se encuentra distribuida a lo ancho de la cordillera Central y la meseta pubenense. El pueblo está 
conformado por los resguardos de Puracé, Coconuco, Paletará, Quintana, Poblazón, Alto del Rey, Guarapamba, que integran la Asociación de Cabildos Indígenas de la Zona Centro, llamada Genaro Sánchez, y a su vez hacen parte del Consejo Regional Indígena del Cauca (CRI) y la Organización Nacional Indígena de Colombia (ONIC).

Un aspecto relevante es la aceptación y la necesidad que tiene la población de ir incluyendo las TIC en sus actividades cotidianas, pues parte de los ideales del actual gobernador del resguardo de Puracé es estimular que la comunidad sea la protagonista de su propio desarrollo: "de acuerdo con sus expectativas e intereses, accediendo, incorporando, fortaleciendo la cosmovisión local con otras miradas externas" (entrevista a Arcadio Aguilar, junio de 2013).

Este proceso se ha ido generando paulatinamente y ha estado signado por la búsqueda constante y colectiva por satisfacer cada vez mejor y más rápidamente su necesidad de comunicación. Solo basta una retrospectiva para establecer cómo se han ido estableciendo acciones de comunicación caracterizada por mecanismos creativos de búsqueda y obtención de información, que facilita las actividades cotidianas. La tabla 1 ilustra las herramientas de comunicación más comunes en el resguardo de Puracé que sirven para enmarcar claramente el uso y apropiación de las TIC desde una perspectiva diacrónica.

Ahora bien, las herramientas tecnológicas mencionadas han ido configurando aspiraciones sociales, satisfacción de necesidades, definición y soluciones de problemas, establecimiento de relaciones sociales, así como tradicionales, económicas y políticas, que no han sido entendidas por ellos como una concatenación autónoma; por lo contrario, han sido entendidas como desarrollos sociales concomitantes a dichas herramientas y las prácticas que derivan de ellas. De ahí la segunda columna de características de la herramienta, la cual busca tipificar cuáles son los elementos componenciales del artefacto y su relación con los demás en un sistema. Así, un computador, por ejemplo, no es un artefacto más (como de repente sí lo puede ser un arma de fuego), es parte de un sistema tecnológico-simbólico que lleva a introducir un valor de significado en un contexto determinado (Lemonnier, 1996). 


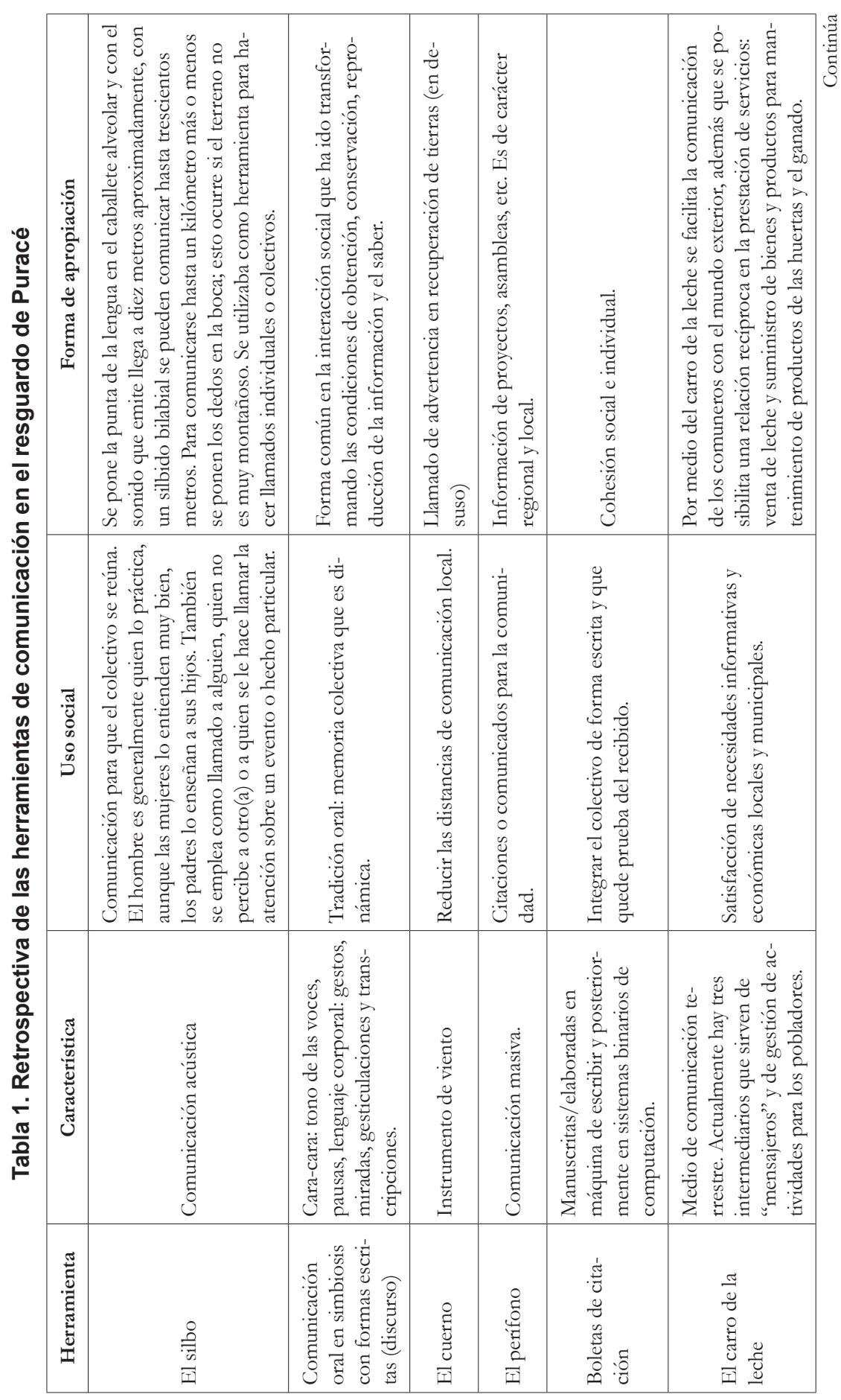




\begin{tabular}{|c|c|c|c|}
\hline 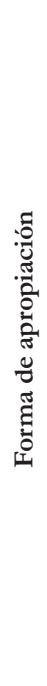 & 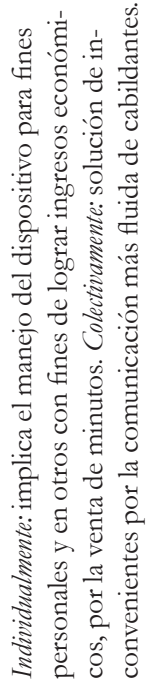 & 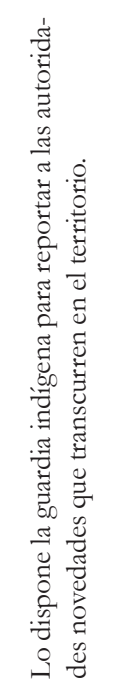 & 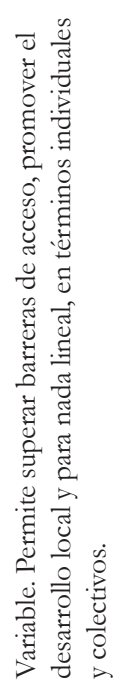 \\
\hline 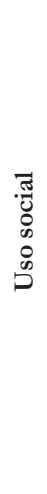 & 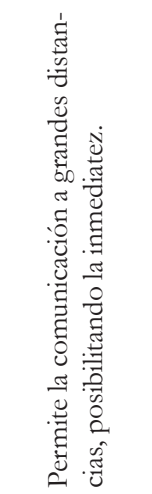 & 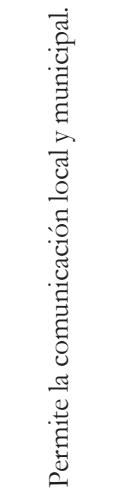 & 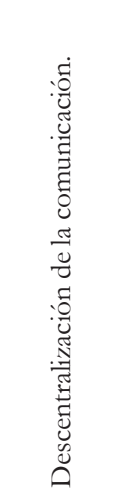 \\
\hline 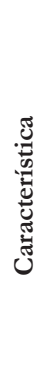 & 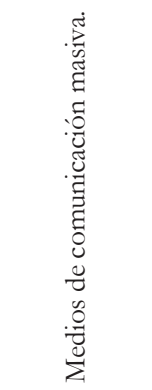 & 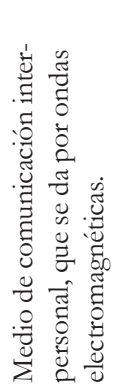 & 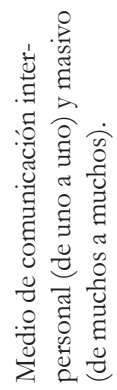 \\
\hline 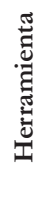 & $\frac{\frac{\pi}{3}}{\frac{\vec{J}}{U}}$ & 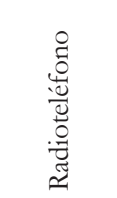 & $\begin{array}{l}\text { 节 } \\
\text { 节 }\end{array}$ \\
\hline
\end{tabular}

Desafíos, Bogotá (Colombia), (26-2): 153-178, semestre // de 2014 
En este escenario toman cuerpo los usos sociales (tercera columna), pues estos hacen referencia a las acciones o las prácticas que el pueblo indígena kokonuco genera en torno a estas herramientas o, lo que es lo mismo, el uso nos remite — según el gobernador del resguardo- a un espacio de análisis, donde el que usa las tecnologías, en general, y las TIC, en particular, es portador de una historia tanto individual como colectiva (Arcadio Aguilar, gobernador del resguardo de Puracé, febrero de 2013). En este sentido quién, cómo, cuándo y, sobre todo, para qué usan estas herramientas entra a formar parte de la apropiación social (cuarta columna), tema que para nada se desvincula con la perspectiva de uso. Esta perspectiva centra su enfoque en los modelos o modos de hacer propios o personaliza las TIC por medio de su uso (Álamo, 2005), o lo que diría Fredy Manquillo: "las formas por medio de las cuales cada uno de nosotros o nosotros hacemos nuestro el 'compu' y lo incorporamos a algunas actividades cotidianas" (entrevista al cabildante, junio de 2013).

Así, el proceso de adaptación y apropiación de las tecnologías termina por convertirse en un uso estratégico local que deriva de unos antecedentes basados en la necesidad de comunicarse. Pero, lejos de ser un dominio exclusivo de las TIC, lo que muestra esta retrospectiva es la coexistencia de variados modos y formas de comunicación que sostienen las relaciones diarias y cotidianas de los comuneros tanto en el interior de sus familias, en relación con el cabildo, como con el mundo exterior.

Efectivamente, se podría decir que en la trayectoria tecnológica que la población ha vivido no se les ha dado la espalda a los medios tradicionales y se ha ido buscando, por el contrario, fortalecer los medios hacia dinámicas masivas en concordancia con procesos locales, regionales y nacionales. Esto se puede traducir en dos ejemplos concretos referidos a otra forma de TIC que se ha ido consolidando en los últimos años en algunos pueblos indígenas del país: la radio y el internet.

Nuestro país, al igual que el Cauca, no estuvo exento de la influencia de internet. Así, a finales de la década de los noventa, la página web del CRIC se encargó de crear un sistema integrado de información y 
comunicación que compactó dentro de un mismo modelo de comunicación todos aquellos medios parte de la cosmovisión indígena de los diversos grupos indígenas que habitan el Cauca. Desde esta perspectiva y en el análisis que me ocupa, la posibilidad de concretar la construcción de la emisora y de visibilizarse en la página del CRIC ha sido un proyecto que no solamente ha ocasionado que sus discursos sobre unidad, territorio y autonomía se constituyan en práctica, sino que también, y como lo manifiesta Fredy Manquillo Comunero, del resguardo, ha posibilitado "vínculos y relaciones" con otros actores sociales de distintas procedencias y entornos sociales.

Enlaces en redes sociales como Facebook o Twitter son otros ejemplos de las plataformas por medio de las cuales se promueven las acciones sociales emprendidas por actores indígenas que denominan minga informativa. En esa minga se promocionan congresos e información de todos los procesos indígenas gestionados en lo local, lo regional y lo internacional.

A los aspectos mencionados se les suma la propuesta del periódico, elemento que si bien se había venido trabajando como medio físico, la ONIC emprendió la tarea de trabajarlo como medio digital y que, al igual que el portal del CRIC, desarrolla programas en pro del fortalecimiento de los planes de vida de los pueblos indígenas en general, tema que ha logrado consolidar espacios físicos (foros, asambleas y cumbres) y digitales (foros, páginas web y blogs) a diferentes escalas. En síntesis, los medios digitales mencionados despliegan un sinnúmero de proyectos políticos comunicativos que vinculan directamente a la población indígena, y entre ellos, a los kokonuco con sus procesos organizativos y sociales-culturales.

Otro lenguaje digital que se ha ido incorporando en las dinámicas del pueblo kokonuco es la emisora Renacer Kokonuco, la cual nace en términos legales bajo la Resolución 071 del $1^{\circ}$ de febrero de 2002, del Ministerio de Telecomunicaciones de Colombia. La emisora refleja el programa de comunicaciones de las comunidades indígenas de la zona 
centro (Genaro Sánchez), por medio de la que dan a conocer procesos organizativos, de gobierno propio, de soberanía alimentaria, de territorio, entre otros. Sale al aire a través de la frecuencia 90.7 FM. Las fuentes informativas que nutren los programas de esta emisora son el CRIC, los cabildos, la alcaldía e internet. Según el gobernador actual del pueblo kokonuco y otros habitantes de la zona, la emisora — desde su inicio- ha constituido una herramienta que poco a poco ha potenciado la participación colectiva y ha hecho evidente un proceso de apropiación de un personal que se ha ido especializando gradualmente y que se ha ido integrando al resto de la población. Tal tema se refleja en la transmisión gratuita de mensajes en aquellas áreas adonde llega la señal, lo que fortalece así un sentido de pertenencia social.

\section{“Gradualidad” y modelos de apropiación social de las TIC en pueblos indígenas: el caso del pueblo kokonuco}

El resguardo de Puracé, que está ubicado en las estribaciones de la cordillera Central hacia las faldas del volcán Puracé, se sobrepone al municipio de Puracé y su cabecera se encuentra ubicada a veintiocho kilómetros de Popayán, capital del departamento del Cauca. Sus límites son: al norte, con el municipio de Totoró; al oriente, a las faldas del volcán Puracé; al sur, con la chorrera de Chirimbolo, y al occidente, con el río San Francisco. El resguardo está conformado geográficamente por las veredas de Crucero-Campamento, Cuaré, 20 de Julio, Chisvar-Hispala, Alto de Anambío, Hato Viejo-Ambiró, Tabío, Patico, Chapio, Pululó y Puracé cabecera (figura 1).

En cuanto a su población, de acuerdo con el censo poblacional elaborado por el Cabildo Indígena de Puracé en 2011, la población del resguardo es de 4446 habitantes, equivalentes a 1143 familias, de los cuales el 3,6\% es analfabeta, el 75,4\% tiene primaria, el 20,8\% ha cursado secundaria y el $0,2 \%$ ha ido a la universidad. El sistema educativo en el territorio está integrado por 10 escuelas y un colegio, los cuales instruyen en los niveles de básica primaria, básica secundaria y bachillerato, desde grado 0 hasta grado 11 . 
Figura 1. Ubicación del resguardo Puracé
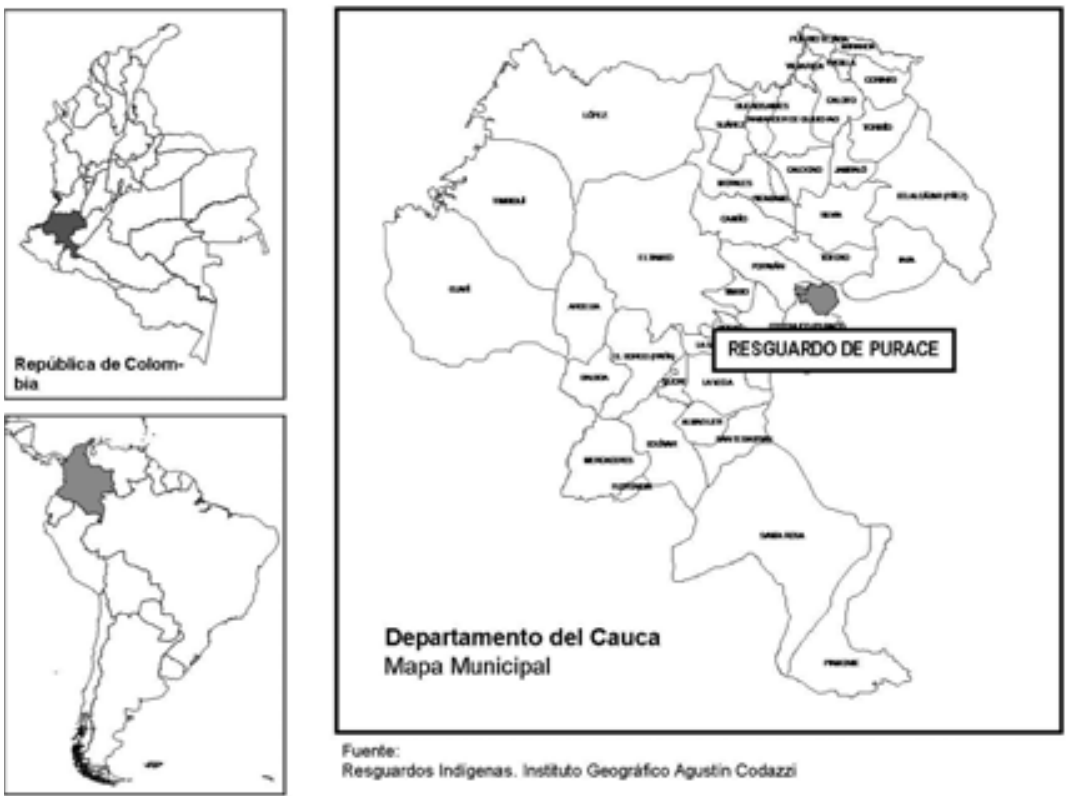

Fuente: Instituto Geográfico Agustín Codazzi.

Ahora bien, los modelos de apropiación en el resguardo se ven representados a través de la categoría de gradualidad, entendida esta como una dimensión temporal que permite evidenciar de qué manera un individuo o grupo social logra apropiar o desechar ciertas técnicas y tecnologías que en algún momento de su existencia se presentan a disposición. Este sentido de gradualidad no opera por sí mismo, sino que está en función de otros componentes vitales como la edad (aspecto generacional), la naturaleza y la pertinencia de la tecnología para sus propósitos de vida. Tanto la gradualidad como la idea de modelo pueden comprenderse más claramente a la luz de un conjunto de antecedentes que afectaron la dinámica de la vida social en el resguardo de Puracé. Veamos esta trayectoria.

Internet en el resguardo ha permitido pensar en crear un portal web que, en primer lugar, sea descentralizado, entendiendo por ello una transferencia de recursos, acceso y conocimientos, un proceso social, cuyo resultado supone un fortalecimiento de las relaciones entre la 
casa del cabildo y las veredas que tendrán cierta autonomía en el diseño de su portal. Si bien la casa del cabildo tendrá la potestad para asumir la concepción del portal del resguardo, las veredas, a su turno, tendrán la potestad de configurar los portales desde su propia apreciación y valoración social, siempre y cuando el interés y vitalidad por el proyecto se sostengan.

Se trata entonces de transferir el poder de decisión de manera que los actores locales actúen como agentes y que cada una de las veredas que componen el resguardo indígena gocen de cierta autonomía y, en consecuencia, tengan un mayor sentido de apropiación social. Así, el modelo de desarrollo local tecnológico que se desea aplicar se basa en un proceso en el cual el flujo de información e interacción con los puntos conectados posibilita dinámicas de trabajo en distintos órdenes: político, social, pedagógico y de discusión sobre ciertos temas comunes, pero estratégicos.

En este sentido, tanto comuneros como cabildantes ven la creación de la página web como un espacio comunitario, en el cual la dirección de acción es como dice el gobernador Arcadio: "de adentro hacia afuera". Con esto quiere decir que la dirección y el direccionamiento sobre uso y apropiación de las TIC parte de identificar no un modelo como tal, sino varios modelos que están en función de componentes asociados con lo social, la percepción de la importancia de lo tecnológico, lo político, entre otros. Se trata de un proceso complejo que por lo pronto permite hablar de tres modelos de percepción que potencialmente vinculan un sentido de apropiación que nos permite ver el papel que las TIC tienen en pueblos indígenas.

En el primer grupo, que llamaremos modelo de apropiación asertivo, se perfila un sentido claro y sostenido de la importancia de las TIC y la sociedad de la información. De este grupo forman parte las veredas Cuaré y 20 de Julio. En el caso de Cuaré, está compuesta por 62 familias, y se percibe una recurrente necesidad de proyectar su cotidianidad en la página web, de expresar "nuestras experiencias reales" (entrevista a Felisa Piso, 52 años, comunera, abril de 2013), sus raíces culturales, la educación de sus hijos, su organización colectiva y participativa, 
sus áreas de trabajo colectivo, sus lugares históricamente sagrados, su economía, su comunicación tradicional (cuerno), el vestido, la alimentación y el medio ambiente. Se trata de elementos que, además de relacionarse con sus usos y costumbres, han posibilitado que los diversos proyectos relacionados con las TIC se articulen con la vereda como un colectivo (figuras 2 y 3 ).

Figura 2. Usos y costumbres para reflejar el portal de Cuaré

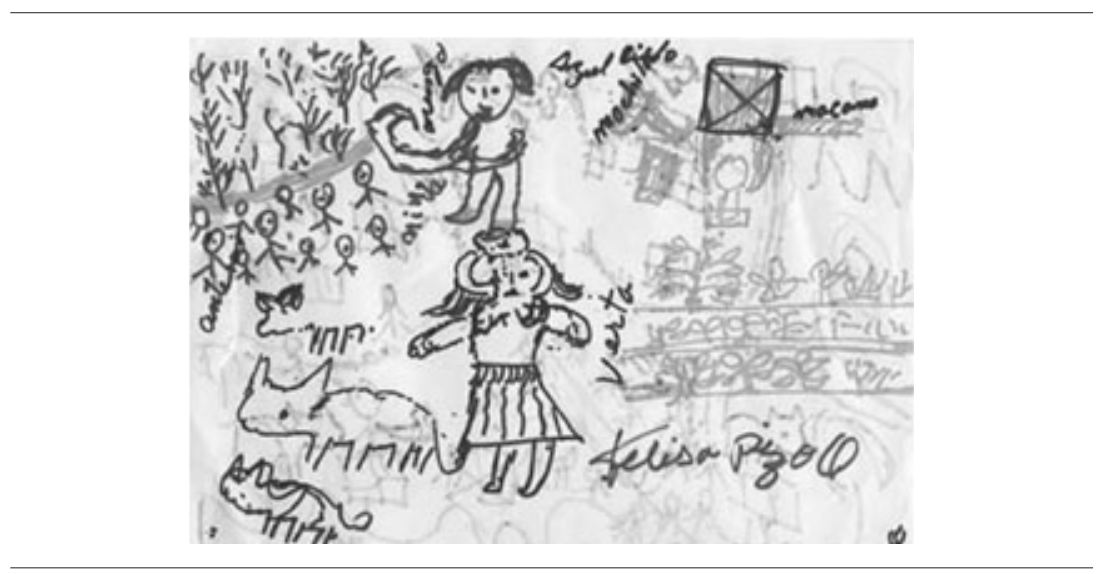

Fuente: Taller de Memoria. Proyecto de Apropiación de las TIC, marzo de 2013.

Figura 3. Escuela y entorno de Cuaré

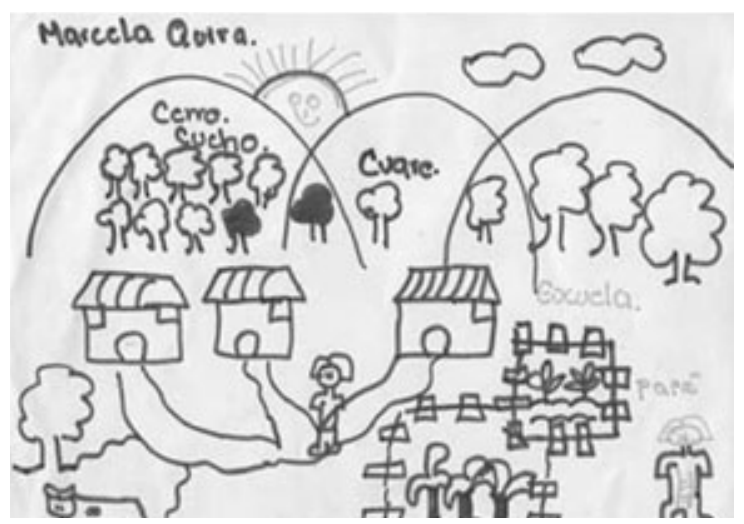

Fuente: Escuela, caminos, agricultura y vivienda. Marcela Quirá. Taller de Memoria, marzo de 2013.

En las visitas realizadas con el objetivo de abordar aspectos socioculturales que se evidenciarían en el diseño del portal en Cuaré, se 
observó que existe una gran expectativa frente a las TIC, sobre todo en el desarrollo del portal, donde se involucraron jóvenes y niños, quienes esperan que del proceso surja una mejor comunicación intrageneracional, pues, como dice Marcela Quirá, "existen muchas historias que no conocemos y nos gustaría escucharlas [lo que permite] digitalizar el pasado y vivir el presente" (cabildante, 10 años, vereda Cuaré, marzo de 2013).

La segunda vereda que forma parte del modelo de apropiación asertivo es 20 de Julio. Se trata de una localidad que nunca ha tenido acceso a internet y que tampoco tiene escuela, computadores o telecentros; pero cuya ausencia ha generado una gran expectativa por sentirse integrados a nivel del resguardo y con el mundo exterior. En la vereda no se han dado programas sociales específicos y su participación ha sido a través del resguardo. Se trata de una comunidad pequeña de treinta familias en la que su principal actividad es la ganadería (doble propósito) y los cultivos de huerta; algunos pobladores se dedican a un trabajo asalariado en veredas vecinas, tanto en territorio de resguardo como por fuera de este.

En general, durante varias sesiones de trabajo con los pobladores de esta vereda, ellos manifestaron el deseo de continuar gradualmente en el proyecto de apropiación de las TIC, pues tienen dos razones de peso. La primera es la aplicación de conocimientos digitales en su vida diaria y la segunda es la solución de problemas concretos, como el desplazamiento constante de sus jóvenes hacia otros lugares para tener acceso a computadores e internet. Cabe destacar que el afán de conjugar TIC, desarrollo local y jóvenes de manera transversal es apostarle a una etapa inicial de alfabetización digital (entrevista a Marcelino Pizo, comunero, junio de 2013).

Un segundo modelo es referido como modelo transicional, y refiere a veredas donde algunos líderes ven la pertinencia del proyecto, pero que una gran mayoría no ve la relación de su vida individual-colectiva con el papel de las TIC. Sugerimos la idea de transicional, porque es posible que, una vez vean los resultados en el primer grupo o en otros escenarios, se pueden decidir por avanzar en el proceso de apropiación, o 
bien sea que dicha intencionalidad se vea disminuida y, posteriormente, abandonada. La primera vereda que forma parte de este modelo es Chisvar-Hispala, ubicada sobre la vía principal que comunica los departamentos del Cauca y el Huila. Está integrada por 71 familias. Los medios de comunicación existentes hoy en día son: las boletas de citación, el celular, el perifoneo e internet, que se consigue fuera de la zona. Algunos de los líderes de la vereda mostraron la intención de participar del proyecto de apropiación de las TIC por su afinidad con ellas. Édgar Marino Piso, cabildante de la zona, es uno de los interesados por las Tic. Él estudió comunicación social durante cinco años en la institución virtual Modern School Inc. y posteriormente estudió en la Universidad del Valle y en la Universidad del Cauca. Actualmente, forma parte de los gestores y locutores de la radio Renacer Kokonuco y es el encargado de trasmitir la información por medio del perifoneo en la vereda.

Un segundo ejemplo de este modelo transicional logramos observarlo en la vereda Vueltas de Patico, donde la población está dividida: los que están cerca de la junta de acción comunal y los que pertenecen a la escuela. Ambos grupos dejan entrever su interés por el acceso, uso y apropiación de las TIC; pero el acceso a la infraestructura está instalado en la escuela. Aquí los docentes foráneos de la ciudad de Popayán, María del Socorro Andrade y Éver Castro, enfocan su trabajo en un sistema educativo indígena, con acciones pedagógicas contextualizadas que le dan relevancia al efecto de cambio climático y a la agroecología. En 2007, se lideró un proyecto en la escuela que se llamó Hacia la soberanía alimentaria y adaptación al cambio climático desde el PEC: la Tulpa, donde los niños, los padres de familia y los docentes se vincularon para darle paso a una dinámica que garantizara la nutrición, la seguridad y la calidad de los alimentos. Dicha dinámica incentivó la creación de un blog (figura 4) y del sitio web http:/ /www. escuelavueltasdepatico.com. Esta comunidad logró gestionar y promover su modelo agro-TIC, a pesar de las dificultades de acceso a internet. En este sentido, el modelo es parcialmente apropiado por docentes y algunos estudiantes; mientras otro grupo de la comunidad ve a la distancia este proceso. 
Figura 4. Blog de la Escuela Vueltas de Patico

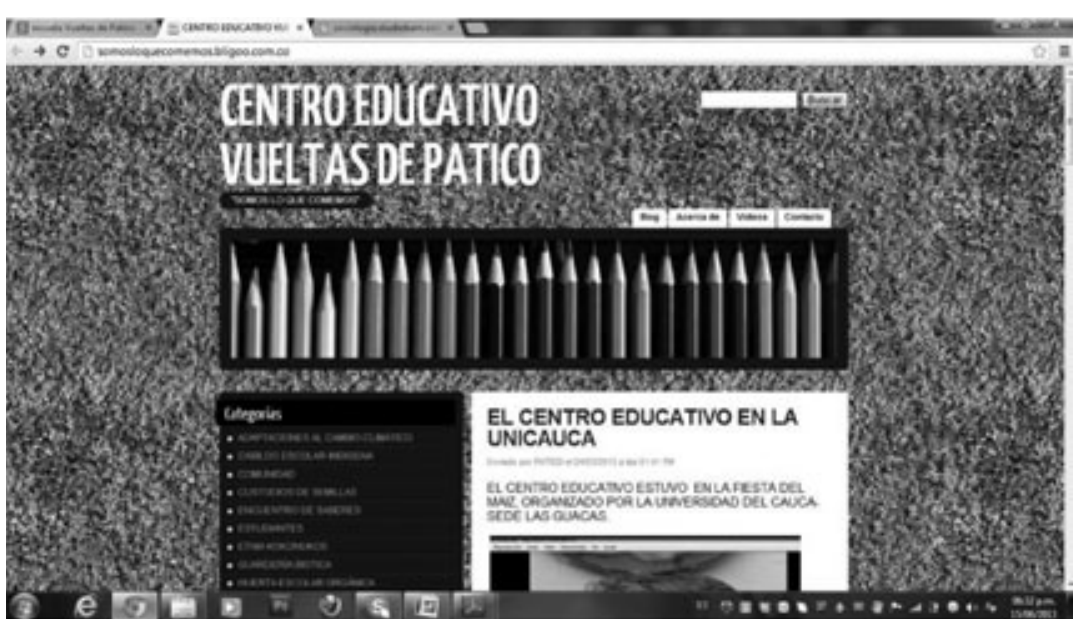

Fuente: elaboración propia a partir de http://somosloquecomemos.bligoo.com.co

El tercer modelo de apropiación, llamado inactivo, alude a aquellas veredas que no han demostrado ningún interés colectivo por avanzar en el uso y apropiación de las TIC, lo que no quiere decir que individualmente, en el plano familiar, no exista esta disposición, sino que la inclinación o interés colectivo no es pertinente para el caso de uso de este tipo de tecnologías. Puede ser que hipotéticamente vean esta relación más en el plano individual que en el colectivo. Por contraste e inverso a Cuaré, los antecedentes individuales sobresalen sobre el interés colectivo.

En aquellos casos más destacados, algunos pobladores argumentan un alto grado de desconocimiento de las TIC y poco interés respecto a querer incorporarse en estos procesos, porque muchas veces las políticas públicas se ejecutan pero con gran mediocridad. En la vereda Campamento, por ejemplo, docentes, profesores y estudiantes hablan del mal estado de los equipos que donó el gobierno. Dicen que fueron equipos reencauchados a los cuales les daban uno o dos años más de utilidad y dejaban de funcionar; equipos que actualmente se encuentran dañados y que instrumentalizan popularmente el eslogan del programa de gobierno "Computadores para educar", por el de "Computadores para arrumar". En general, para algunos de los ha- 
bitantes de estas veredas, las TIC e internet, en particular, aún no son aliadas en sus vidas cotidianas.

Para concluir este apartado, si bien hemos podido comprobar la importancia de las TIC para el pueblo kokonuco, esta herramienta presenta diversos modelos y apropiaciones que muestran procesos de transformación comunicacional local.

\section{A manera de conclusión}

Los datos etnográficos destacan la forma en la que el pueblo indígena kokonuco va incursionando en los nuevos espacios digitales. En tal proceso tecnológico influye no solamente la tecnología, sino que además se configuran redes en las que se ponderan las relaciones de comunicación social en los ámbitos local, nacional y regional; relaciones que en buena medida evidencian la manera en la que se va configurando la práctica tecnológica en el pueblo indígena y que plantea así diversas posibilidades de uso y apropiación.

En esta medida, pensar en el terreno ganado por las poblaciones indígenas en los espacios digitales de la contemporaneidad es pensar en dos vías: la primera, reflexionar en la materialización de dicho proceso, el cual se ha llevado a cabo a través de emisoras indígenas y de diversos portales elaborados teniendo en cuenta varios ejes articuladores que vinculan derechos colectivos en términos de autonomía colectiva. En este sentido, tanto el pueblo indígena kokonuco como las organizaciones Genaro Sánchez, el CRIC, la ACIN y la ONIC han ido incorporando el uso de las TIC en diversas actividades que implican para ellos acceder a infraestructura, información y conocimientos, aspectos vitales para sus procesos de resistencia indígena.

Partiendo de la idea mencionada en el párrafo anterior, la segunda vía se refiere a la experiencia particular del pueblo kokonuco frente a las TIC. Las especificidades de uso y apropiación social van conectadas con las demandas sociales, pues construyen y deconstruyen identidades y relaciones sociales en espacios digitales. Tal hecho supone el surgimiento de modelos de apropiación en diferentes niveles y prácticas de 
participación que dependen de la cohesión social de este mismo pueblo (gradualidad). Al respecto identifiqué tres, que no son los únicos y que seguramente el tiempo dirá de qué manera se hacen sostenibles o no: un modelo inactivo, otro transitorio y un modelo donde el uso y apropiación de las TIC se hace más efectiva. La idea de estos tres modelos no es unilineal ni pretende ser evolutiva; sencillamente son formas de emergencia en cuanto al relacionamiento entre tradición indígena, uso de las TIC y su pertinencia para fortalecer a la primera.

Para finalizar, considero que para que las TIC sean sostenibles por esta población, especialmente por las veredas que muestran interés en participar, deben darse unas condiciones mínimas referidas al acompañamiento de dichos procesos y su relación con la presencia de proyectos estatales y no gubernamentales, los cuales adelantan procesos pedagógicos con distintos actores. El fenómeno de la apropiación de las TIC en este artículo avizora una cualificación de modelos en poblaciones indígenas que tanto los indígenas como los investigadores están llamados a responder.

\section{Referencias}

Álamo, Ó del (2005). Hacia la apropiación social de las nuevas tecnologías en los procesos de desarrollo. Gobernanza. Revista Internacional para el Desarrollo Humano (28). Recuperado de http://www.redelaldia.org/ IMG/pdf/0448.pdf.

Ardévol, E. (2003). La cibercultura: un mapa de viaje; aproximaciones teóricas para un análisis cultural de Internet. Aportaciones al Seminario Pensar la Cibercultura, Antropología y Filosofía del Nuevo Mundo. Recuperado de http://eardevol.files.wordpress.com/2008/10/eardevol_cibercultura.pdf

Appadurai, A. (2001). Dislocación y diferencia en la economía cultural global. En La modernidad desbordada. Buenos Aires: Fondo de Cultura Económica.

Bertalanffy, L. (2007). Teoría general de sistemas. México: Fondo de Cultura Económica.

Cabrera, J., Moriones, G. y Quinchoa, J. (2003). La tecnología en Colombia: algunos apuntes teórico-metodológicos desde la antropología. s. d. 
Castell, M. (1999). La era de la información: economía, sociedady cultura (vol. 1, La sociedad red). México: Siglo XXI.

Escobar, A. (2005). Bienvenidos a Cyberia: notas para una antropología de la cibercultura. Revista Estudios Sociales (22), 15-35.

Gow, D. (2010). Replanteando el desarrollo: modernidad indígena e imaginación moral. Bogotá: Editorial Universidad del Rosario.

Latour, B. (2005). Reensamblar lo social: una introducción a la teoría del actor-red. Buenos Aires: Manantial-SRL.

Lemonnier, P. (1996). Technology. En Encyclopedia of social and cultural anthropology. London: Routledge.

Marcus, G. (2001). Etnografía en/sistema mundo: surgimiento de la etnografía multilocal. Alteridades (24), 102-127.

Medina, M. (2003). La cultura de la tecnociencia. En B. Marieta (Ed.), Nuevas tecnologías y cultura. México: Anthropos.

Ministerio de Comunicaciones de la República de Colombia (2008). Plan nacional de tecnologías de la información y la comunicación. Recuperado de http://www.mintic.gov.co/portal/604/w3-channel.html

Moriones, G. (2011). Procesos tecno-organizativos: usos y resignificaciones de las tecnologías de la información y la comunicación (TIC) en espacios organizativos de la parcialidad indígena de Puracé. Pueblo Kokonuco, Universidad del Cauca.

Mazabuel, Q. (2002). Plan de vida resguardo indígena Puracé, Cabildo Indígena de Puracé Departamento del Cauca. s. d.

Rapport, N. y Overing, J. (2007). Social and cultural anthropology: The key concepts. Oxon: Routledge.

Rodríguez, C, y Gazi, J. (2007). La poética de la radio indígena en Colombia. En Ya no es posible el silencio. Bogotá: Friedrich Ebert Stiftung. Recuperado de http://www.c3fes.net/docs/poetica_radio_rodriguez_elgazi.pdf

Sandt, J. v. d. (2012). Detrás de la máscara del reconocimiento. Popayán: Universidad del Cauca.

Tocancipá-Falla, J. (2013). Repensando los modelos, representaciones y cartografías conceptuales: aproximaciones desde la antropología. En C. Castrillón, C. Hernández y J. Tocancipá-Falla (Eds.), Modelo, diseños y radio enlaces. Popayán: Universidad del Cauca.

Ulloa, A. (2012). Reconfiguraciones conceptuales, políticas y territoriales en las demandas de autonomía de los pueblos indígenas en Colombia. Bogotá: Tabula Rasa.

Woolgar, S. (1996). Technology. En A. Kuper y J. Kuper (Eds.), The social science encyclopaedia. London: Routledge. 